\title{
A Comparative Analysis of Rare Sternalis Muscles: A Case Report
}

\author{
Adegbenro Omotuyi John Fakoya ${ }^{1 *}$, Jessica Heymans ${ }^{2}$, Lennis N. Colón Rivera ${ }^{2}$, Brian Acevedo Fuentes ${ }^{2}$, \\ Abayomi Gbolahan Afolabi ${ }^{1}$, Thomas McCracken ${ }^{1}$ \\ ${ }^{1}$ Department of Anatomy, University of Medicine and Health Sciences, Basseterre, St. Kitts and Nevis; ${ }^{2}$ Medical Student, \\ University of Medicine and Health Sciences, Basseterre, St. Kitts and Nevis
}

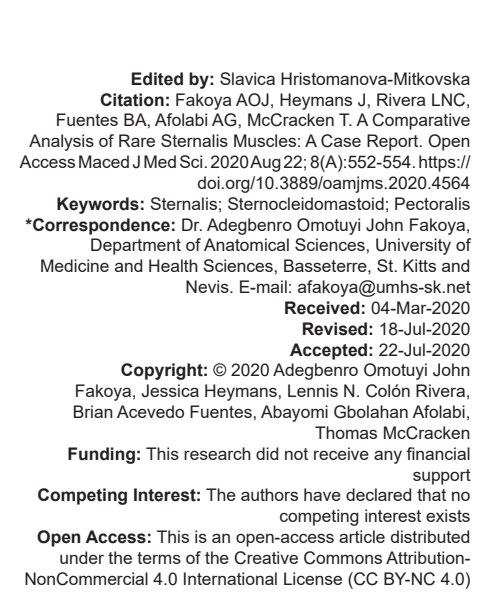

Abstract

The sternalis muscle is a rare variant in the anterior chest wall located anterior to the vertical muscle. It was found in two cadavers during routine cadaveric dissection of 20 bodies. This finding provided an opportunity to perform a comparative anatomical analysis between a unilateral sternalis muscle on a female and a bilateral sternalis muscle on a male. Having a better understanding of the anatomical variants can be extremely useful, precisely to avoid misdiagnosing tumors and to assist in landmark identification during surgeries.

\section{Introduction}

The sternalis muscle is an uncommon muscle found in the anterior chest wall of the thoracic region. It is a vertical muscle that lies parallel to the sternum and anterior to the pectoralis major [1]. It is also known as "parasternalis" and "rectus sterni" muscle, due to its anatomical positions. The muscle can either be a bilateral or a unilateral variant. It usually originates from the sternum and the inferior border of the clavicle and can display variable insertion points [2].

The function of sternalis muscle is still unknown. It is assumed that it assists in the movement of the shoulder and/or in the elevation of the lower chest wall [3]. Studies have mentioned the ability of the muscle to confuse physicians in misdiagnosing abnormal mass during a mammogram screening. Its surgical benefit includes flap in reconstruction surgery of the head and neck, anterior chest wall, and breast [1].

Here, we will be reporting and comparing two sternalis muscles encountered during cadaveric dissections.

\section{Case Presentation}

A routine dissection of the anterior chest wall of 20 cadavers by medical students at the Anatomy Laboratory, University of Medicine and Health Sciences, St. Kitts and Nevis revealed an uncommon muscle, the sternalis muscle, in two cadavers, and variations between these muscles were observed.

The first cadaver was an 82-year-old female whose sternalis muscle was unilateral and located only on the left. It was attached to the sternum and was found to be a continuation of her sternocleidomastoid muscles which joined to form this muscle. The sternalis muscles traveled medially along the anterior chest wall producing a horizontal branch over her pectoralis muscles. After this, it continued its course, turning slightly to the right and curving inferiorly to the cadaver's pectoral region (Figure 1).

Comparatively, the second cadaver was a 56-year-old male. Similarly, the muscle also arose from a union between her sternocleidomastoid muscles and was attached to the sternum. Unlike the previous 


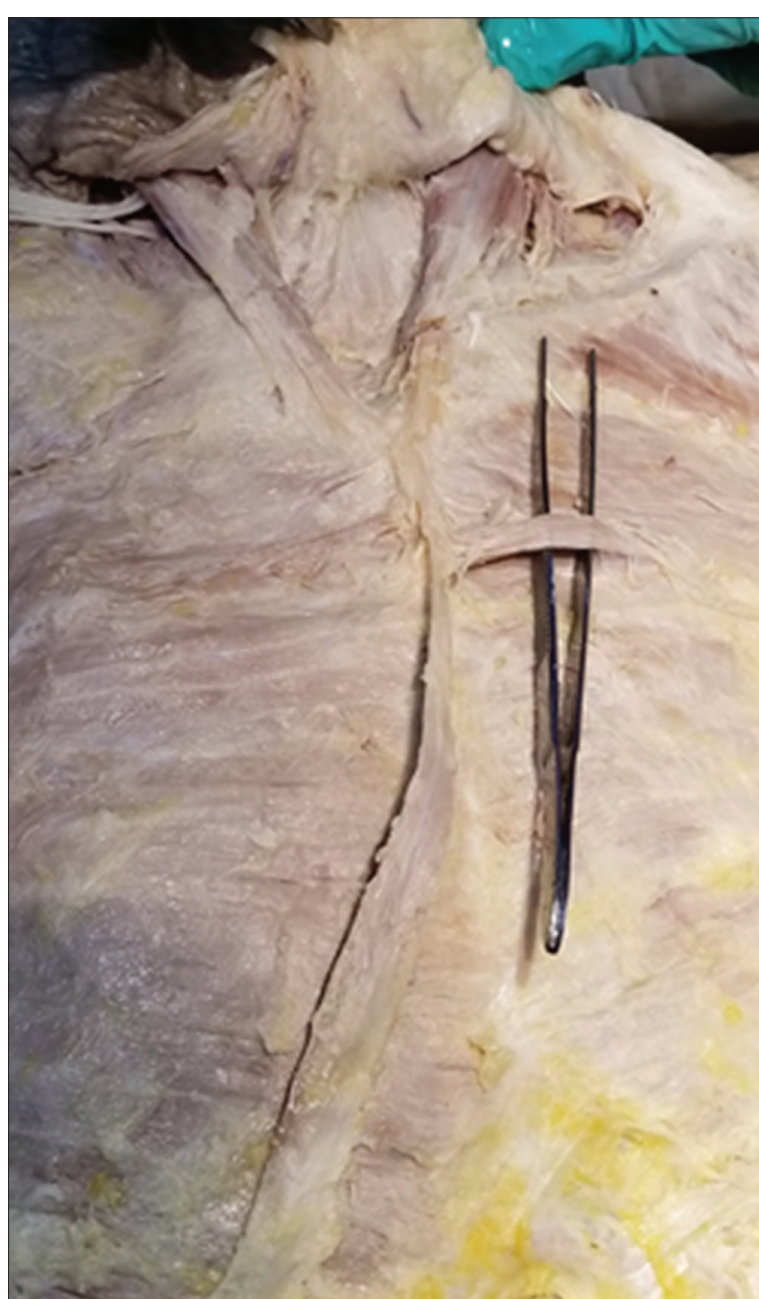

Figure 1: Unilateral sternalis muscle of the cadaver of an 82-year-old female

dissection, the sternalis muscle on this cadaver was bilateral and had a varied trajectory. Although both began its course traveling medially, the sternalis muscle on this cadaver had multiple originating branches before it reached the mid-pectoral region to divide bilaterally and continued to the end of its course (Figure 2).

\section{Discussion}

The sternalis muscle is a very rare presentation in the anterior chest wall that is common to females. Sarıkçıoglu reported the presence of three sternalis muscles in a male cadaver (one on the right and two on the left side) [2]. Unilateral sternalis is more frequently seen $(4.5 \%)$ than a bilateral sternalis $(1.7 \%)$. In this case, the female cadaver had a unilateral sternalis muscle, while the male cadaver had a bilateral sternalis muscle. It usually appears as a flat muscle with varying thickness and sometimes with tendinous insertions in the anterior chest wall. It is anterior and perpendicular to the pectoralis major muscle and lateral to the sternum [3]. The innervation for the sternalis muscle can be easily damaged during dissection

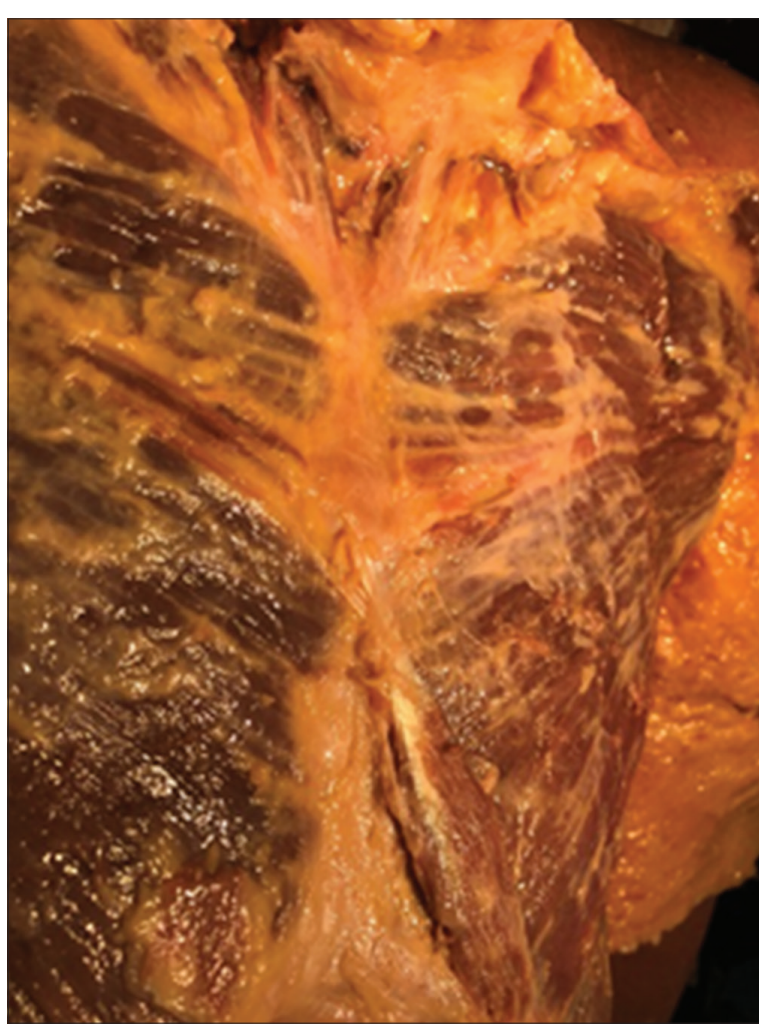

Figure 2: Bilateral sternalis muscle of the cadaver of a 56-year-old male

or surgery. A study reported that $55 \%$ of the cases were innervated by branches of thoracic nerves, while $43 \%$ were by the intercostal nerves [4]. Furthermore, the blood supply for this muscle is primarily by the internal thoracic artery and sometimes by the thoracoacromial artery [4]. In our dissection of the muscles of both cadavers, the innervation and origin of the muscles could not be determined.

There are several theories on the origin of the sternalis muscle. Some postulated that the sternalis muscle is derived from the hypaxial masses of the sternocleidomastoid muscle or even the remnants of the panniculus carnosus muscle sheet [3]. Similarly, there also various theories on the function of the muscle, though it suggests based on the orientation of its fibers that it may help in elevation of the chest wall or shoulder joint $[2,3]$. However, the muscle is used as a pedicle flap or flap microvascular anastomosis during reconstructive surgery of the anterior chest wall, breast, head, and neck [1]. The only claimed clinical significance is the potential to cause a misdiagnosis of breast tumors in mammograms, showing an abnormal bulge [5]. Moreover, the use of magnetic resonance imaging or computed tomography allows better identification of this muscle if present.

\section{Conclusion}

Although the sternalis muscle is rare, physicians and clinicians may need to be aware and familiar with the sternalis muscle. This knowledge may prevent the 
misdiagnoses of tumors in routine mammograms. It is a great advantage to notice the existence of this muscle in cadaveric dissections so it can be better studied and evaluated. In this case, we had the opportunity to compare the presence of a unilateral sternalis muscle in a female cadaver and a bilateral sternalis muscle in a male cadaver.

\section{References}

1. Raikos A, Paraskevas G, Tzika M, Faustmann P, Triaridis S, Kordali $\mathrm{P}$, et al. Sternalis muscle: An underestimated anterior chest wall anatomical variant. J Cardiothorac Surg. 2011;6(1):73. https://doi.org/10.1186/1749-8090-6-73

PMid:21575244

2. Sarıkçıoglu L. Three sternalis muscles associated with abnormal attachments of the pectoralis major muscle. Int J Exp Clin Anat 2008;2:67-71. https://doi.org/10.2399/ana.08.067

3. Chaudhary D, Gopal D, Rymbai D. Rectus sternalis muscle a rare variant. Int J Trend Sci Res Dev. 2019;3(2):299-301. https:// doi.org/10.31142/ijtsrd21348

4. Katara P. Unilateral rectus sternalis muscle: Rare but normal anatomical variant of anterior chest wall musculature. J Clin Diagn Res. 2013;7(12):2665-7. https://doi.org/10.7860/ jcdr/2013/7379.3726

PMid:24551605

5. Loukas M, Bowers M, Hullett J. Sternalis muscle: A mystery still. Folia Morphol. 2004;63(2):147-9.

PMid:15232768

Author Queries???

AQ5: Kindly provide abstract subheadings 